\title{
A $\beta$ Protofibrils Possess a Stable Core Structure Resistant to Hydrogen Exchange ${ }^{\dagger}$
}

\author{
Indu Kheterpal, ${ }^{\ddagger}$, Hilal A. Lashuel, ${ }^{\S, \|}$ Dean M. Hartley," Thomas Walz, ${ }^{\perp}$ Peter T. Lansbury Jr.," and \\ Ronald Wetzel*,* \\ Graduate School of Medicine, R221, University of Tennessee, 1924 Alcoa Highway, Knoxville, Tennessee 37920, \\ Center for Neurologic Diseases, Brigham and Women's Hospital and Department of Neurology, Harvard Medical School, \\ 65 Landsdowne Street, Cambridge, Massachusetts 02139, and Department of Cell Biology, Harvard Medical School, \\ 240 Longwood Avenue, Boston, Massachusetts 02115
}

Received October 2, 2003; Revised Manuscript Received October 24, 2003

\begin{abstract}
Protofibrils are transient structures observed during in vitro formation of mature amyloid fibrils and have been implicated as the toxic species responsible for cell dysfunction and neuronal loss in Alzheimer's disease (AD) and other protein aggregation diseases. To better understand the roles of protofibrils in amyloid assembly and Alzheimer's disease, we characterized secondary structural features of these heterogeneous and metastable assembly intermediates. We chromatographically isolated different size populations of protofibrils from amyloid assembly reactions of $\mathrm{A} \beta(1-40)$, both wild type and the Arctic variant associated with early onset familial $\mathrm{AD}$, and exposed them to hydrogen-deuterium exchange analysis monitored by mass spectrometry (HX-MS). We show that HX-MS can distinguish among unstructured monomer, protofibrils, and fibrils by their different protection patterns. We find that about $40 \%$ of the backbone amide hydrogens of $\mathrm{A} \beta$ protofibrils are highly resistant to exchange with deuterium even after 2 days of incubation in aqueous deuterated buffer, implying a very stable, presumably H-bonded, core structure. This is in contrast to mature amyloid fibrils, whose equally stable structure protects about $60 \%$ of the backbone amide hydrogens over the same time frame. We also find a surprising degree of specificity in amyloid assembly, in that wild type $\mathrm{A} \beta$ is preferentially excluded from both protofibrils and fibrils grown from an equimolar mixture of wild type and Arctic mutant peptides. These and other data are interpreted and discussed in terms of the role of protofibrils in fibril assembly and in disease.
\end{abstract}

Amyloid fibrils are the primary protein component of neuritic plaques found in the brains of Alzheimer's disease $(\mathrm{AD})^{1}$ patients and are associated with more than 20 other amyloid diseases (3). In $\mathrm{AD}$, the amyloid plaques are composed primarily of the $\mathrm{A} \beta$ peptide, a 39-43 residue proteolytic product of a transmembrane protein called amyloid precursor protein (APP) (4). All four types of mutation associated with early onset familial $\mathrm{AD}$ are linked to increased production and/or deposition of $\mathrm{A} \beta$ peptides in the brain (5). Although some studies find that the classically stained $\mathrm{A} \beta$ plaque burden does not correlate perfectly with

\footnotetext{
Supported in part by NIH R01AG18927 to R.W. and NIH R01AG08470 to P.T.L.

* To whom correspondence should be addressed. Phone: (865) 5449168. Fax: (865) 544-9235. E-mail: rwetzel@mc.utmck.edu.

University of Tennessee.

$\S$ These authors have contributed equally to the work presented in this publication.

"Brigham and Women's Hospital and Department of Neurology, Harvard Medical School.

${ }^{\perp}$ Department of Cell Biology, Harvard Medical School.

${ }^{1}$ Abbreviations: HX-MS, hydrogen-deuterium exchange mass spectrometry; ESI-MS, electrospray ionization mass spectrometry; SEC, size exclusion chromatography; AD, Alzheimer's disease; PBS, phosphate-buffered saline; Tris, tris(hydroxymethyl)aminomethane; TFA, trifluoroacetic acid; HFIP, 1,1,1,3,3,3-hexafluoro-2-propanol.
}

$\mathrm{AD}$ progression (6), there does appear to be a correlation between disease and the ratio of soluble to insoluble $\mathrm{A} \beta$ in the brain (7). One attractive hypothesis for resolving the contrasting genetic and pathological trends is that the toxic agent in $\mathrm{AD}$ is not the extracellular amyloid plaque but rather another aggregated form of $\mathrm{A} \beta$, possibly a prefibrillar oligomeric assembly intermediate.

Nonfibrillar oligomeric forms of $\mathrm{A} \beta$ clearly exist. Oligomeric forms collectively referred to as protofibrils are typically transiently observed when relatively high, nonphysiological concentrations of monomeric $\mathrm{A} \beta$ are incubated in vitro to generate amyloid fibrils $(8,9)$. Oligomeric forms of $\mathrm{A} \beta$ have also been characterized in cell cultures $(10,11)$. Exogenous and endogenous $\mathrm{A} \beta$ protofibrils are cytotoxic (11, $12)$, a property they share with oligomeric assembly intermediates of other amyloidogenic proteins (13). Striking evidence in support of a role for protofibrils in AD comes from a recently described early onset familial AD mutation in the $\mathrm{A} \beta$ peptide itself (E22G) called the Arctic mutation (14). The Arctic mutation in $\mathrm{A} \beta$ ( $\left.\mathrm{A} \beta_{\mathrm{ARC}}\right)$ has an increased propensity for forming protofibrils as compared to wild type $\mathrm{A} \beta$ ( $\left.\mathrm{A} \beta_{\mathrm{WT}}\right)$, suggesting that this mutation predisposes individuals to early-onset $\mathrm{AD}$ due to the formation of relatively long-lived, toxic protofibrils (14). 
Recently, Lashuel and co-workers (15) identified conditions that permit the preparation, separation (by size exclusion chromatography (SEC)), and biophysical characterization of stable protofibrillar species of $\mathrm{A} \beta$. Under these conditions, electron microscopic (EM) examination of the protofibrils formed by $\mathrm{A} \beta(1-40)_{\mathrm{ARC}}$ revealed several morphologies, including (a) relatively compact spherical particles roughly 4-5 nm in diameter, (b) annular pore-like protofibrils (6-9 nm o.d. and 1.5-2 i.d.), (c) large spherical particles $18-25 \mathrm{~nm}$ in diameter, and (d) short filaments with chainlike morphology. Interestingly, protofibrils formed from an equimolar mixture of $\mathrm{A} \beta_{\mathrm{WT}}$ and $\mathrm{A} \beta_{\mathrm{ARC}}$, a biologically relevant mixture of the two proteins that may model the situation in heterozygous patients, are more stable than those formed by $\mathrm{A} \beta_{\mathrm{ARC}}$ and $\mathrm{A} \beta_{\mathrm{WT}}$ alone (15). To better understand their roles in both assembly of amyloid fibrils and $\mathrm{A} \beta$ cytotoxicity, it is important to further characterize the structural properties of these protofibrils.

Due to their large size, heterogeneous morphology, and poor solubility, amyloid fibrils and protofibrils have so far resisted high-resolution structure determination. The metastability of protofibrils presents an additional impediment. There are, however, intermediate resolution methods that can provide insights into the structures of monomers, protofibrils, and fibrils and to the structural relationships among these states. In particular, hydrogen-deuterium exchange (HX) provides significant insight into structure by determining the pattern and extent of hydrogen bonding within the aggregate (1). HX methods take advantage of the fact that exchange of backbone amide protons involved in H-bonded secondary structures such as $\alpha$-helices and $\beta$-sheets, and/or those buried in core structure, is substantially slower in comparison to backbone protons in non-H-bonded regions such as loops and random coil structures. HX has been widely used in conjunction with mass spectrometry (MS) to study protein structure and dynamics $(16,17)$. HX-MS techniques have recently been extended to study the structures of amyloid fibrils $(1,2,18,19)$. Using these methods, we found that $\mathrm{A} \beta$ amyloid fibrils consist of a very rigid core structure, most likely an H-bonded $\beta$-structure, which involves only 19 of the $39 \mathrm{~A} \beta$ backbone amides $(1,2)$.

In this paper, we extend these methods to characterize the structure in the soluble oligomeric intermediates formed in $\mathrm{A} \beta_{\mathrm{ARC}}$ and mixed $\mathrm{A} \beta_{\mathrm{ARC}} / \mathrm{A} \beta_{\mathrm{WT}}$ fibrillization reactions. Details of protofibril structure may provide critical insights into the mechanism of amyloid fibril formation and its role in the mechanism of pathogenesis as well as the design and development of $\mathrm{AD}$ therapeutics.

\section{MATERIALS AND METHODS}

Protofibril Synthesis. Protofibrils were prepared from chemically synthesized $\mathrm{A} \beta_{\mathrm{WT}}(1-40)\left(\mathrm{NH}_{2}\right.$-DAEFRHDSGYEVHHQKLVFFAEDVGSNKGAIIGLMVGGVV-COO$\mathrm{H})$ and $\mathrm{A} \beta_{\mathrm{ARC}}(1-40$ Arctic $(\mathrm{E} 22 \mathrm{G}))$ monomers purchased as trifluoroacetic acid (TFA) salts from the Biopolymer Facility at Brigham and Women's hospital. The detailed procedure for the preparation of $\mathrm{A} \beta$ protofibrils has been described previously (15). Briefly, lyophilized $\mathrm{A} \beta$ monomer (either Arctic or an equimolar mixture of wt and Arctic) was dissolved in $1 \mathrm{mM} \mathrm{NaOH}$ and then diluted to $100 \mu \mathrm{M}$ in $0.2 \times$ PBS, $\mathrm{pH} 7.4$ followed by incubation at room temperature for 16-24 h. Large, insoluble particles (consisting of $15-20 \%$ of the total $\mathrm{A} \beta$ monomer) were removed by 5 min centrifugation at $13000 \mathrm{~g}$ and hereafter will be referred to as pellet. Protofibrils and small soluble $\mathrm{A} \beta$ species in the supernatant were separated using a Superdex 75 HR (Amersham Pharmacia) SEC column equilibrated with $5 \mathrm{mM}$ Tris- $\mathrm{HCl}, \mathrm{pH} 7.4,70 \mathrm{mM} \mathrm{NaCl}$. The protein was eluted at a flow rate of $0.5 \mathrm{~mL} / \mathrm{min}$. The size exclusion chromatogram consisted of a void volume peak corresponding to oligomeric species of different sizes and morphologies (protofibrils) and a relatively sharp peak corresponding to species of smaller hydrodynamic radii, predominantly monomeric $\mathrm{A} \beta$ (low molecular weight; LMW) (15). The presence of small amounts of rapidly equilibrating oligomers (dimers, trimers, and tetramers) in the LMW peak cannot be ruled out. The oligomeric and LMW peaks were split into several fractions in the order of their elution (PF1-PF3 and LMW1 and LMW2) and stored at $4{ }^{\circ} \mathrm{C}$. The pellet and the protofibril fractions were spotted on the grids for EM and also expressshipped on ice for HX experiments.

Hydrogen Exchange. SEC fractions were stored on ice after their isolation, and HX experiments were initiated within $24 \mathrm{~h}$ and completed within another $48 \mathrm{~h}$. A 250-500 $\mu \mathrm{L}$ aliquot of protofibril fractions was placed in a YM-10 microcon tube (Millipore) and centrifuged at $14000 \mathrm{~g}$ at $3{ }^{\circ} \mathrm{C}$. Since highly concentrated protofibril samples readily form fibrils, the centrifugation time $(30-50 \mathrm{~min})$ was selected to prevent their concentration on the filter by more than 5-fold. Each concentrated sample was diluted with $\mathrm{D}_{2} \mathrm{O}$ and centrifuged at $3{ }^{\circ} \mathrm{C}$, and the process was repeated one to two times until the $\mathrm{H}_{2} \mathrm{O}$ content reached $<2 \%$. The filter was then inverted and centrifuged at $1000 \mathrm{~g}$ for $3 \mathrm{~min}$ to collect the sample in a fresh centrifuge tube, and the sample was analyzed for deuterium content after being adjusted to an equivalent monomer concentration of $15-20 \mu \mathrm{M}$. The average time to prepare each sample was $2.5 \pm 0.5 \mathrm{~h}$. This method of HX sample preparation maintained the integrity of the protofibril sample while removing salts and introducing $\mathrm{D}_{2} \mathrm{O}$. After $\mathrm{HX}$ analysis, samples were held on ice for up to an additional $24 \mathrm{~h}$ and then were analyzed by EM. Importantly, EMs of the HX analyzed samples confirmed that the protofibril fractions had not progressed to mature fibrils during analysis (see Results and Figure 1).

The pellets formed during the preparation of $\mathrm{A} \beta_{\mathrm{ARC}}$ protofibrils (after $16 \mathrm{~h}$ of incubation at $37{ }^{\circ} \mathrm{C}$ ) were either incubated at $37{ }^{\circ} \mathrm{C}$ for an additional $24 \mathrm{~h}$ to generate mature fibrils or were stored on ice until $\mathrm{HX}$ experiments were carried out. Pure $\mathrm{A} \beta_{\mathrm{WT}}(1-40)$ fibrils were generated by disaggregating monomer using TFA and hexafluoroisopropanol (HFIP) (Pierce) followed by incubation at $37^{\circ} \mathrm{C}$ in $1 X$ PBS for 5-7 days (20).

For HX experiments on fibrils and pellets, aggregates were collected by centrifugation, washed once with $2 \mathrm{mM}$ Tris$\mathrm{HCl}$ buffer, and resuspended in D-Tris-DCl buffer as described in detail previously (1). For HX experiments on monomers, $\mathrm{A} \beta_{\mathrm{ARC}}$ and $\mathrm{A} \beta_{\mathrm{WT}}$ were disaggregated in TFA and HFIP as stated previously, lyophilized, then dissolved in 2 $\mathrm{mM}$ D-Tris-DCl buffer, and immediately analyzed as described previously (1).

ESI-MS. The HX equipment and protocol have been described previously $(1,2)$. Briefly, the Z electrospray source of a Quattro II (Micromass) triple quadrupole mass spec- 

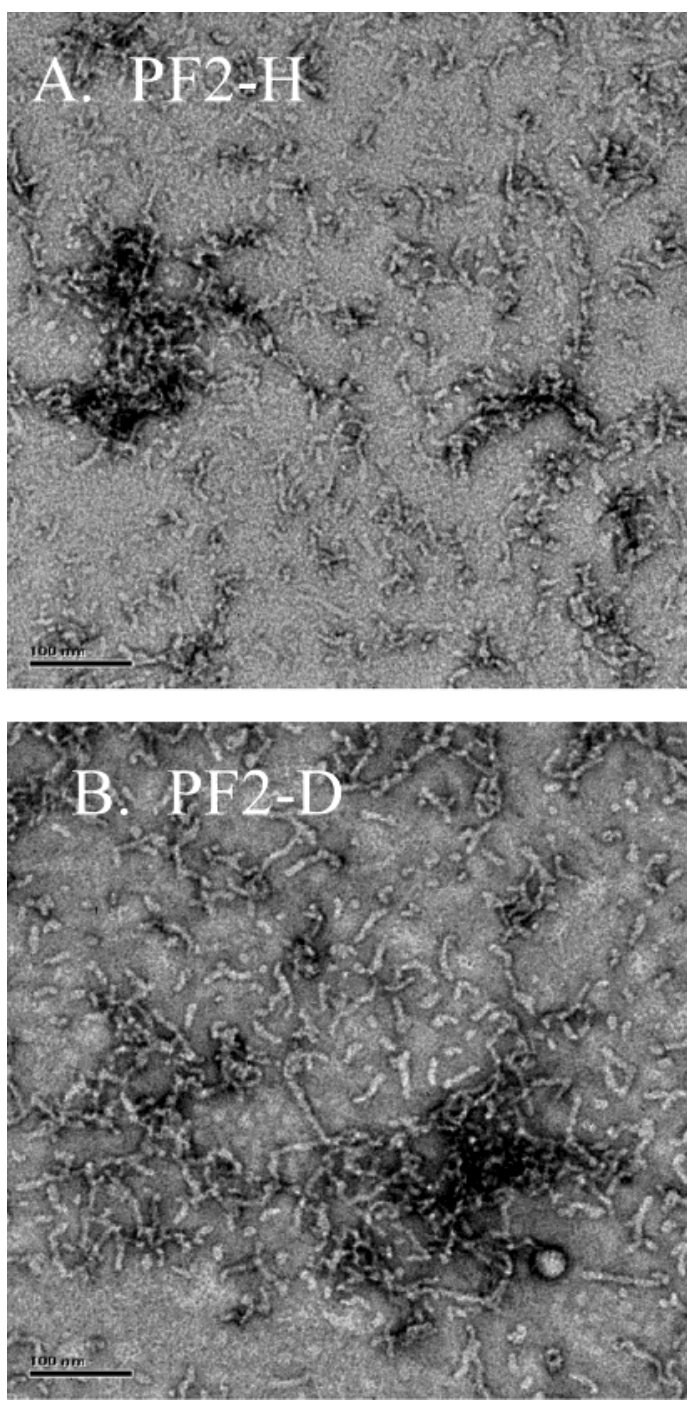

FIGURE 1: EM of negatively stained images of protofibril fraction before and after HX experiments. Both images depict samples obtained from a 1:1 equimolar mixture of $\mathrm{A} \beta_{\mathrm{ARC}}$ and $\mathrm{A} \beta_{\mathrm{WT}}$ monomer purified on a Superdex 75 SEC column. (A) Freshly separated protofibril fraction PF2. (B) Same PF2 fraction after HX experiments. Scale bars are equal to $100 \mathrm{~nm}$.

trometer was modified with a Valco $\mathrm{T}$ union. The sample solution was infused through one arm of the $\mathrm{T}$ where it was mixed with quenching and dissolving solvent (50:50:0.5 [v/v/ v] $\mathrm{H}_{2} \mathrm{O} / \mathrm{CH}_{3} \mathrm{CN} / \mathrm{HCOOH}$ ) being pumped through the second arm of the $\mathrm{T}$. The flow rates used for sample and solvent infusion were 0.5 and $9.5 \mu \mathrm{L} / \mathrm{min}$, respectively. The mixed solution was then electrosprayed into the MS using the third arm of the T. Data were obtained in the multichannel acquisition mode with mass/charge $(\mathrm{m} / \mathrm{z})$ ranging from 650 to 1150 at a rate of $2 \mathrm{~s} / \mathrm{scan}$.

Data Analysis. The mass spectra were smoothed using the Savitzky Golay method implemented in the MassLynx program (Micromass) and deconvoluted to obtain centroid and peak area using the Pearson VII equation in PeakFit (Systat) program. Molecular masses were calculated as $\left\{(m / z) z-\left[z+\left(z\left(\% \mathrm{D}_{2} \mathrm{O}\right)\right)\right]\right\}$ using centroids of the unresolved isotopic envelops for the +5 and +6 charge states of the $\mathrm{A} \beta$ peptide as described in detail previously (2). Resulting mass values derived from both +5 and +6 charge states were averaged to obtain the molecular masses. The percentage of $\mathrm{D}_{2} \mathrm{O}$ in the final solvent mixture being sprayed into the MS was kept constant at $10 \%$ for all the experiments presented here.

Electron Microscopy. Fibrillar and protofibrillar samples of $\mathrm{A} \beta$ were diluted 2 -fold with protein buffer $(10 \mathrm{mM}$ Tris$\mathrm{HCl}, \mathrm{pH} 7.4,75 \mathrm{mM} \mathrm{NaCl}$ ) prior to adsorption to glowdischarged carbon-coated copper grids. Grids were washed with four drops of buffer and stained with two drops of freshly prepared $0.75 \%$ uranyl formate (Pfaltz and Bauer, Waterbury, CT). Specimens were inspected with a Philips Tecnai 12 electron microscope operated at $120 \mathrm{kV}$, and images were taken at a nominal magnification of $52000 \times$ using low dose procedures.

\section{RESULTS}

Handling of $A \beta$ Protofibrils. The metastability of $\mathrm{A} \beta$ protofibrils presents particular technical challenges to their structural analysis. Figure 1 demonstrates that under the conditions we have developed, there is no significant difference in the structural properties, as discerned by EM, of protofibrils before and after HX analysis. In particular, protofibril preparations post-analysis exhibit no trace of mature amyloid fibrils.

In previous $\mathrm{HX}$ studies of mature fibrils, it was possible to rapidly remove buffer salts and introduce $\mathrm{D}_{2} \mathrm{O}$ by centrifugation $(1,2)$. However, protofibrils are not pelleted by low-speed centrifugation, while pelleting of protofibrils by high-speed centrifugation transforms them into fibrils. Consequently, a gentler, but also more time-consuming, ultrafiltration protocol (Materials and Methods) was used to conduct the buffer exchange of protofibrils. The disadvantage of this protocol is that early time points $(<2 \mathrm{~h})$ of the exchange kinetics cannot be obtained.

Finally, the lack of stability and heterogeneity of the protofibril population renders complete correction of the HX data for artifactual exchange into backbone amide protons very difficult (2). In the work reported here, therefore, correction for artifactual exchange is confined to the rapidly exchanging protons.

$H X$ in $A \beta_{A R C}$ Aggregates. The extent of exchange of backbone amide protons to deuterons was measured for the four SEC fractions PF1-PF3 (protofibrils) and LMW1 (monomer rich fraction), plus $\mathrm{A} \beta_{\mathrm{ARC}}$ monomer and fibrils. Figure $2 \mathrm{~A}$ shows representative spectra for each one of these samples after $\sim 2 \mathrm{~h}$ of exchange. For reference, the dashed lines in the top panel correspond to fully protonated, and fully deuterated, $\mathrm{A} \beta_{\mathrm{ARC}}$ monomers, the lower and upper mass limits for this peptide. The $m / z$ ratio measured after $\mathrm{HX}$ for fibrils and fractions PF1-PF3 and LMW1 fall between these two limits.

Although the HX mass spectrum of fraction LMW1 is symmetric, suggesting a homogeneous population of molecules, the mass spectra of fractions PF1-PF3 are broad and asymmetric (Figure 2A). These qualities are probably due to the latter fractions being heterogeneous mixtures of different protofibril structures (ref 15; Figure 1), as well as the likelihood that fractions PF2 and PF3, due to incomplete SEC separation, may contain smaller $\mathrm{A} \beta$ species. The asymmetric peaks in the mass spectra of PF1-PF3 could be reasonably fitted using two peaks, hereafter referred to as $\mathrm{A}$ and $\mathrm{B}$ populations, with peak $\mathrm{B}$ corresponding to a more complete incorporation of deuterium. Thus, there are 

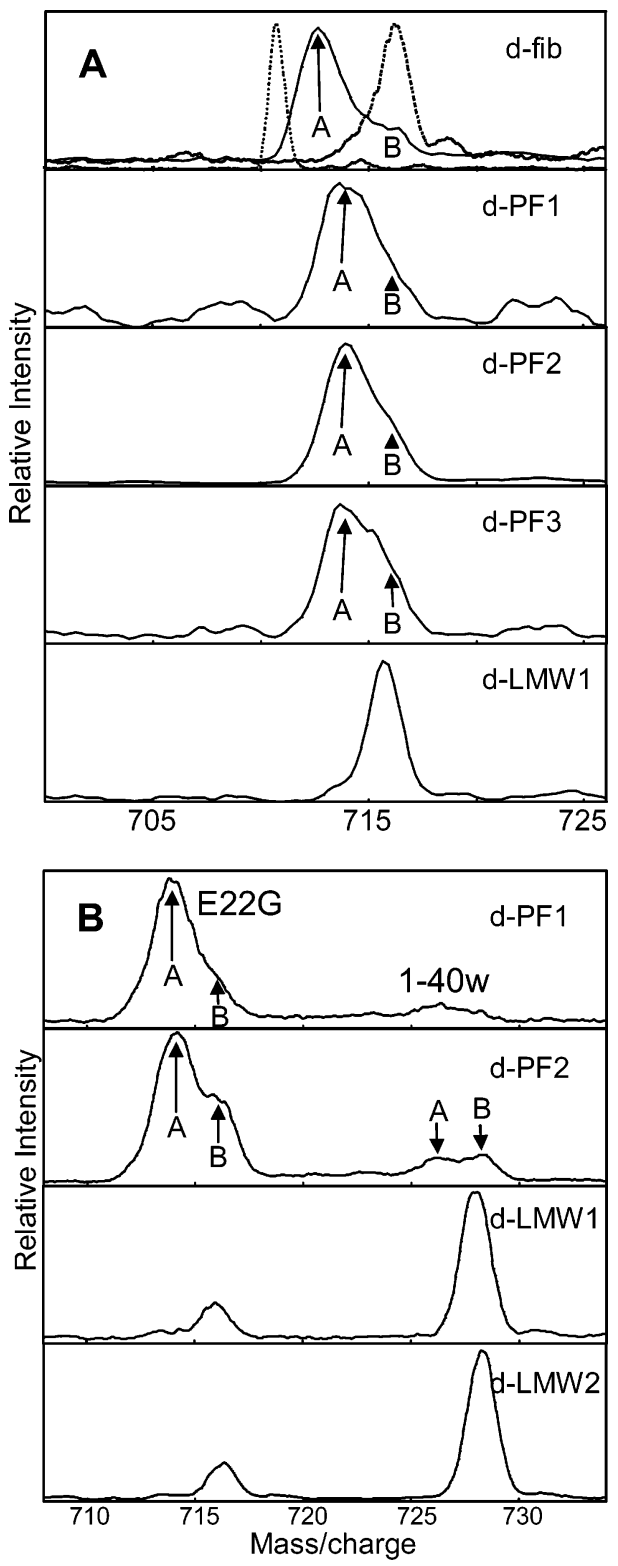

FIGURE 2: (A) ESI-MS showing +6 charge state of deuterated $\mathrm{A} \beta$ ARC fibrils and deuterated $\mathrm{A} \beta_{\mathrm{ARC}}$ protofibrils (PF1-PF3) and LMW fraction after $2 \mathrm{~h}$ of incubation in a deuterated buffer. Mass spectra of protonated and deuterated $\mathrm{A} \beta_{\mathrm{ARC}}$ monomer are shown as dashed lines in the top panel for comparison and reference. (B) ESI-MS showing +6 charge state of deuterated protofibril fractions and LMW fractions from 1:1 mix of $\mathrm{A} \beta_{\mathrm{ARC}}$ and $\mathrm{A} \beta_{\mathrm{WT}}$ after $2 \mathrm{~h}$ of incubation in a deuterated buffer. Two populations deconvoluted using curve fitting are labeled as A and B.

at least two distinct molecular environments for $\mathrm{A} \beta$ present in each of the fractions PF1-PF3. These two peaks could also be due to two different structural states within a protofibril. For example, the peptide molecules at the ends of the protofibrils will be expected to exchange more than those in the inner core structure. This would also be true for mature amyloid fibrils, but the smaller protofibrils are expected to contain a larger relative population of surfaceassociated monomers and hence possess a much larger relative population of peak $\mathrm{B}$. Another possibility for the source of peak B would be the dissociation of protofibrils into monomers, which are amenable to complete exchange.

The centroids from the deconvoluted peaks were used to calculate the number of deuteriums exchanged in the two structural groups in fractions $\mathrm{PF} 1-\mathrm{PF} 3$. These measured numbers of deuteriums were corrected for rapidly backexchanging side-chain and terminal protons $(1,2)$, and the corrected data were plotted with respect to HX time (Figure $3 \mathrm{~A})$. Data-fitted dashed lines for deuterated $\mathrm{A} \beta_{\mathrm{ARC}}$ monomer and deuterated $\mathrm{A} \beta_{\mathrm{WT}}$ fibrils are included for reference. ${ }^{2}$ The $\mathrm{HX}$ into $\mathrm{A} \beta_{\mathrm{ARC}}$ fibrils clearly overlaps with that in $\mathrm{A} \beta_{\mathrm{WT}}$ fibrils, suggesting that the fibrils of the Arctic mutant are very similar in structure to those of wild type $\mathrm{A} \beta$. As previously described for $\mathrm{A} \beta_{\mathrm{WT}}$ fibrils (1), the HX pattern for $\mathrm{A} \beta$ ARC fibrils is complex, including a small amount of material with a deuterium content corresponding to that for unrestricted exchange into monomeric $\mathrm{A} \beta$ (population $\mathrm{B}$ in Figure 2A), in addition to a large amount of material exhibiting a significant exchange protection (population A in Figure 2A). To varying degrees, similar complex patterns are observed in the HX of SEC fractions PF1-PF3 (Figure $2 \mathrm{~A})$. In the simplest case, the LMW fraction, consisting of $\mathrm{A} \beta$ species of smaller hydrodynamic radii, exchanges $\sim 28$ protons in $\sim 2 \mathrm{~h}$, with no further exchange with time, behavior similar to that observed for monomeric $\mathrm{A} \beta$ (Figure $3 \mathrm{~A})$.

The earlier eluting SEC fractions PF1-PF3 all exhibit both the $\mathrm{A}$ and the $\mathrm{B}$ populations characterized by different degrees of exchange protection. In all three fractions, the B population exhibits the same amount of exchange, 29 deuteriums. Interestingly, the proportion of population B in each fraction appears to increase as the hydrodynamic radius of the fractionating species decreases (Figure 2A). In contrast, population $\mathrm{A}$ in fractions $\mathrm{PF} 1-\mathrm{PF} 3$ (Figure 2A) from the $\mathrm{A} \beta_{\text {ARC }}$ assembly is significantly protected from exchange, incorporating 16 deuteriums by the first time point of $\sim 2 \mathrm{~h}$ with a gain of only two more deuteriums over the next 25 h. The metastability of protofibrils raises the possibility that the exchange protection observed for these fractions might not be due to the protofibrils themselves but to fibrils that developed from the protofibrils during the exchange reaction. However, EM images (Figure 1) clearly show that the protofibril structure has not changed during the HX experiment.

The data presented above show that mature fibrils, PF1PF3, and LMW 1 fractions all exchange differently. Overall, the amount of exchange into the protected A population of protons increases as the size decreases. Thus, fibrils exchange the least, protofibril fractions PF1-PF3 exchange more, and fraction LMW1 (the last eluting, smallest $\mathrm{A} \beta_{\mathrm{ARC}}$ species) exchanges the highest number of protons for deuterons. Exchange into LMW1 is similar, but not identical, to HX into monomeric $\mathrm{A} \beta$ (the significance of this relatively minor difference in exchange amplitude needs to be investigated). Thus, HX-MS can be used to differentiate between monomer, protofibrils, and fibrils. Further, it appears that protofibrils contain a very stable core structure, highly resistant to exchange, just like fibrils $(1,2)$.

$H X$ in $A \beta_{W T} / A \beta_{A R C}$ (1:1 Equimolar Mix) Aggregates. Protofibrils formed from a mixture of $\mathrm{A} \beta_{\mathrm{WT}}$ and $\mathrm{A} \beta_{\mathrm{ARC}}$ are

${ }^{2}$ The kinetics data on $\mathrm{A} \beta_{\mathrm{WT}}$ fibrils were obtained as described previously (1) with the exception that the final solvent composition contained $10 \% \mathrm{D}_{2} \mathrm{O}$. The completely corrected deuterium content after $140 \mathrm{~h}$ of exchange under the conditions used here is $19.3 \pm 0.7$ as compared to $20.1 \pm 1.4$ reported earlier (2). 

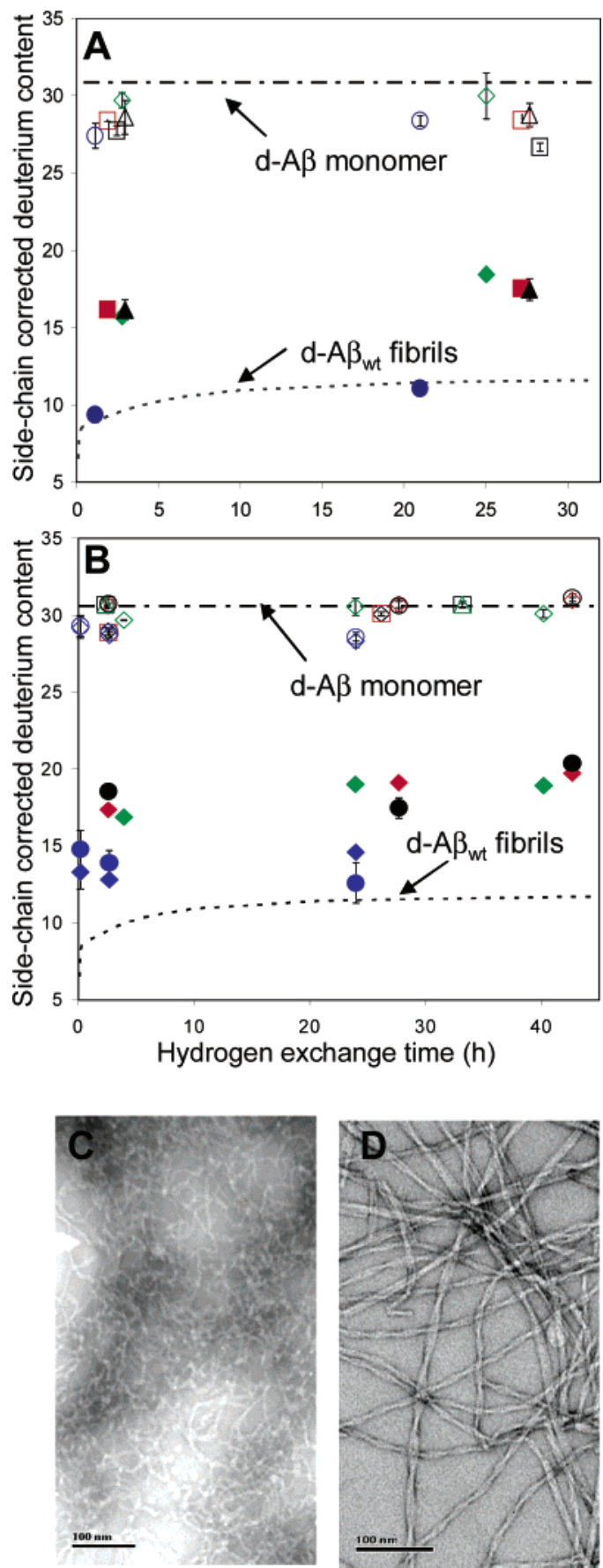

FIGURE 3: (A) Side-chain corrected deuterium content in $\mathrm{A} \beta_{\text {ARC }}$ fibrils (blue circles) and fractions PF1 (green diamonds), PF2 (red squares), PF3 (black triangles), and LMW1 (black squares). (B) Side-chain corrected deuterium content in $\mathrm{A} \beta_{\mathrm{WT}} / \mathrm{A} \beta_{\mathrm{ARC}}$ pellet (blue diamonds, $\mathrm{A} \beta_{\mathrm{ARC}}$; blue circles, $\mathrm{A} \beta_{\mathrm{WT}}$ ), protofibril fraction $\mathrm{PF} 1$ (green diamonds, $\mathrm{A} \beta_{\mathrm{ARC}}$ ), protofibril fraction PF2 (red diamonds, $\mathrm{A} \beta_{\mathrm{ARC}}$; black circles, $\mathrm{A} \beta_{\mathrm{WT}}$ population), LMW1 (black diamonds, $\mathrm{A} \beta_{\mathrm{ARC}}$; red squares, $\mathrm{A} \beta_{\mathrm{WT}}$ ), and LMW2 (black squares, $\mathrm{A} \beta_{\mathrm{ARC}}$; green triangles, $\mathrm{A} \beta_{\mathrm{WT}}$ ). The closed and open symbols represent the deuterium exchange into populations $\mathrm{A}$ and $\mathrm{B}$, respectively, from Figure 2. The top and bottom dashed lines represent the deuterium level observed for $\mathrm{A} \beta_{\mathrm{ARC}}$ monomer and $\mathrm{A} \beta_{\mathrm{WT}}$ fibrils, respectively. (C) $\mathrm{EM}$ of negatively stained images of $\mathrm{A} \beta_{\mathrm{ARC}}$ pellet obtained after $16 \mathrm{~h}$ of incubation at room temperature. (D) EM of negatively stained images of $\mathrm{A} \beta_{\mathrm{ARC}}$ mature fibrils. Scale bars are equal to $100 \mathrm{~nm}$.

unusually stable, persisting in solution for $>60 \mathrm{~h}$ as compared to $24 \mathrm{~h}$ for those formed by $\mathrm{A} \beta_{\mathrm{ARC}}$ alone (15). To analyze the structures of these stabilized species, soluble oligomers
Table 1: Summary of the Number of Deuteriums Measured under the Indicated Conditions

\begin{tabular}{lcc}
\hline \multicolumn{1}{c}{ sample } & $\begin{array}{c}\text { no. of deuteriums } \\
\text { after } 2 \pm 1 \mathrm{~h} \text { of } \\
\text { exchange time }^{a}\end{array}$ & $\begin{array}{c}\text { no. of deuteriums } \\
\text { after } 24 \mathrm{~h} \text { of } \\
\text { exchange time }^{a}\end{array}$ \\
\hline $\begin{array}{l}\text { protected fraction in protofibrils, } \\
\text { population } \mathrm{A}^{b}\end{array}$ & $17.2 \pm 0.8$ & $18.1 \pm 1.2$ \\
$\begin{array}{l}\text { exposed fraction in protofibrils, } \\
\text { population B }\end{array}$ & $29.6 \pm 1.4$ & $29.8 \pm 1.9$ \\
$\begin{array}{l}\text { pellets, population A } \\
\text { fibrils, population A } \\
\text { monomers }\end{array}$ & $13.2 \pm 1.2$ & $13.4 \pm 1.5$ \\
& $9.3 \pm 0.5$ & $11 \pm 0.4$ \\
\hline
\end{tabular}

${ }^{a}$ All values were corrected for fast exchanging side-chain and terminal protons. ${ }^{b}$ Since exchange into all protofibril fractions is equivalent (Figure 3), an average value for each sample is reported here.

and LMW species generated from a 1:1 $\mathrm{A} \beta_{\mathrm{WT}} / \mathrm{A} \beta_{\mathrm{ARC}}$ mix were separated into four SEC fractions, which were then characterized by HX-MS.

Figure $2 \mathrm{~B}$ presents representative mass spectra from the four fractions (PF1, PF2, LMW1, and LMW2) after $\sim 2 \mathrm{~h}$ of HX time. Since the molecular weight of $\mathrm{A} \beta_{\mathrm{WT}}$ is $72 \mathrm{Da}$ higher than that of $\mathrm{A} \beta_{\mathrm{ARC}}$, peaks of $\mathrm{m} / \mathrm{z}, 710-717$ correspond to exchange into $\mathrm{A} \beta_{\mathrm{ARC}}$, and peaks of $\mathrm{m} / \mathrm{z}, 722-730$ correspond to exchange into $\mathrm{A} \beta_{\mathrm{WT}}$. These spectra exhibit a number of interesting features. First, even though an equimolar ratio of the $\mathrm{A} \beta_{\mathrm{WT}}$ and $\mathrm{A} \beta_{\mathrm{ARC}}$ peptide was used to prepare the fibrillization reaction, only a very small quantity of $\mathrm{A} \beta_{\mathrm{WT}}$ is present in the oligomeric fractions PF1 and PF2, which are consequently dominated by the more aggressively amyloidogenic $\mathrm{A} \beta_{\mathrm{ARC}}$ peptide. Correspondingly, smaller species LMW1 and LMW2 contain primarily $\mathrm{A} \beta_{\mathrm{WT}}$ peptide and relatively little $\mathrm{A} \beta_{\mathrm{ARC}}$. As seen for the intermediates in fibril assembly of $\mathrm{A} \beta_{\mathrm{ARC}}$ alone (Figure $2 \mathrm{~A}$ ), the mass spectra of PF1 and PF2 are broad and asymmetric; the unresolved isotopic envelopes for both $\mathrm{A} \beta_{\mathrm{WT}}$ and $\mathrm{A} \beta_{\mathrm{ARC}}$ peptides are reasonably fitted using two peaks (labeled as $\mathrm{A}$ and $\mathrm{B}$ in Figure 2B), and the centroids of these peaks were used to calculate the number of deuteriums incorporated. ${ }^{3}$

Figure $3 \mathrm{~B}$ presents a plot of a side-chain corrected number of deuteriums measured in $\mathrm{A} \beta_{\mathrm{WT}} / \mathrm{A} \beta_{\mathrm{ARC}}$ fractions with respect to HX time. Even though only a very small amount of $\mathrm{A} \beta_{\mathrm{WT}}$ is incorporated into the higher MW fractions, the amount of exchange into both Arctic and wild type peptides is essentially equivalent in each fraction. Moreover, the amount of exchange into these fractions is very similar to that in the corresponding fractions from $\mathrm{A} \beta_{\mathrm{ARC}}$ assembly (Figure 3A). Thus, the population A of fractions PF1 and PF2 exchanged 17 amide protons after $2 \mathrm{~h}$ and only two more protons over the next $40 \mathrm{~h}$. Exchange into population B of PF1 and PF2, and in fractions LMW1 and LMW2 as well, remained constant at $\sim 30$, the same amount of exchange that occurs in fresh samples of monomeric $\mathrm{A} \beta$.

The pellets isolated from early time points of the fibril formation reactions of $\mathrm{A} \beta_{\mathrm{ARC}}$ alone and the $\mathrm{A} \beta_{\mathrm{ARC}} / \mathrm{A} \beta_{\mathrm{WT}}$ mixture were also analyzed using HX-MS (Table 1; Figure $3 \mathrm{~B}, \mathrm{C})$. Interestingly, exchange into this pelleted material is slightly higher than for mature fibrils but lower than for protofibrils (Table 1). Thus, these pellets (Figure 3C) appear

${ }^{3}$ Multiple peaks may be present for $\mathrm{A} \beta_{\mathrm{ARC}}$ in fractions PF3 and PF4 and for $\mathrm{A} \beta_{\mathrm{WT}}$ in fraction PF1, but the signal-to-noise is very poor for confident identification and molecular weight determination. 
to represent intermediate structures between soluble protofibrils and mature amyloid fibrils (Figure 3D). EM studies revealed less ordered fibrillar structures for the pellets relative to mature fibrils (formed by further incubation of the pellets at $37^{\circ} \mathrm{C}$ ), which exhibited more extended, and tightly associated, fibrils. The periodical twisting observed in the mature fibrils, but not in the pelleted material, is also consistent with higher ordered fibrillar structures. As seen in all other oligomeric states described here, these pellets exhibit protected (peak A) and unprotected (peak B) populations. In the $\mathrm{A} \beta_{\mathrm{ARC}} / \mathrm{A} \beta_{\mathrm{WT}}$ mixture, $70 \%$ of the pelleted $\mathrm{A} \beta_{\mathrm{WT}}$ is fully exchanged within the first hour, suggesting that it is not incorporated into the growing fibril structure.

\section{DISCUSSION}

Understanding the assembly of amyloid fibrils will provide important insight into human disease. Recreating amyloid fibril assembly under physiological conditions in vitro and elucidating the underlying mechanisms, however, also raise formidable, intriguing challenges. The waxing and waning of various protofibrillar assemblies in the process of amyloid fibril formation suggests that these structures may lie on the pathway for fibril formation and may even be required intermediates (21). It is also possible, however, that these structures lie off-pathway, as transient reservoirs for $\mathrm{A} \beta$ molecules that leave, and later re-enter, the productive fibril formation pathway. Are protofibrils on- or off-pathway intermediates in amyloid assembly? What is the nature of protofibril structure, and how does that structure relate to the structures of mature fibrils? How might the structures of protofibrils mediate neurodegenerative disease?

The HX data reported here suggest that while protofibrils possess some degree of stable $\mathrm{H}$-bonded structure; the amount of that structure is significantly lower than in mature fibrils (Table 1). The degree of stability of this protected structure is somewhat surprising. One might have expected protofibrils to possess no rigid, protective structure at all, at best perhaps displaying populations of amide hydrogens that exchange slowly but eventually completely. We find, however, that protofibrils possess a core structure involving $40 \%$ of the backbone amide protons that are as stably protected as the amide protons in the highly stable core of mature amyloid fibrils. Circular dichroism studies on these samples indicate the presence of $\beta$-sheet rich structures (data not shown), consistent with previous findings (22). Thus, the most likely source of this highly protective structure is a set of H-bonds in $\beta$-sheet elements within the protofibril. The impression of incrementally increasing degrees of highly protective structure, from monomers to protofibrils to fibrils, is supporting evidence for an on-pathway role for protofibrils and for the hypothesis that protofibrils possess atomic-level structural elements that are retained in the mature fibril.

Other HX data presented here support this model. The progressive increase in the proportion of fully exchanged population $\mathrm{B}$, as the size of the assemblies decreases from fibrils to small protofibrils to monomer, also suggests precursor-product relationships among this series of assemblies. Also, the surprising preferential exclusion of $\mathrm{A} \beta_{\mathrm{WT}}$ from the fibril population formed from a 1:1 mix of $\mathrm{A} \beta_{\mathrm{WT}}$ and $\mathrm{A} \beta_{\mathrm{ARC}}$ is mirrored in the $\mathrm{A} \beta_{\mathrm{WT}} / \mathrm{A} \beta_{\mathrm{ARC}}$ ratios of protofibrils. The small amount of $\mathrm{A} \beta_{\mathrm{WT}}$ present in protofibrils from the mixed reaction is nonetheless mechanistically important as suggested by the relative stability of protofibrils from a 1:1 mix reaction (15). Thus, the $\mathrm{A} \beta_{\mathrm{WT}}$ population in these protofibrils is a minor component that nonetheless affects assembly rates; in essence, $\mathrm{A} \beta_{\mathrm{WT}}$ appears to be a competitive inhibitor of $\mathrm{A} \beta_{\mathrm{ARC}}$ fibril assembly. $\mathrm{A} \beta_{\mathrm{WT}}$ might accomplish this as a monomer, or as an independent protofibrillar assembly, that docks to the growing fibril/protofibril but is incapable of locking into place to propagate the fibril $(23$, 24). The dramatic preferential incorporation of $\mathrm{A} \beta_{\mathrm{ARC}}$ observed in both protofibrils and fibrils in the 1:1 mix assembly reaction suggests a higher degree of specificity in amyloid fibril formation than previously suspected and has general implications for amyloid fibril structure, structural dynamics, and assembly.

There appear to be at least two general ways in which the amount of protective $\mathrm{H}$-bonded structure might increase in the progression of protofibrils to fibrils. One possibility is that protofibrils possess a similar folded $\mathrm{A} \beta$ conformation to that appearing in fibrils but with fewer of the intermolecular H-bonds seen in the latter; the amount of protective, internal H-bonding might increase in a kind of global consolidation of structure underlying the protofibril to fibril transition. Another possibility is that the internal H-bonded structure within $\mathrm{A} \beta$ in the protofibrils is more or less identical to that in fibrils and that the increase in total protective structure observed in the transition is due to new H-bonds formed at the interfaces between protofibril units when they assemble into fibrils. These same two possibilities also exist for the novel, immature fibrils, isolated here from early assembly time points by centrifugation, which exhibit protection intermediate between protofibrils and mature fibrils. These and other open questions about protofibril and fibril structure and the relationship between them should be testable by further HX experiments.

There are a number of hypotheses for how aggregates might be toxic to cells in various amyloid disorders. Some proposed mechanisms depend on functional activities of aggregates that one imagines should be associated with specific structures; among these are the ability to insert into and depolarize membranes (25) and the ability to specifically recruit other proteins into the aggregate (26). Other proposed mechanisms, such as the ability of aggregates to interact with, and eventually overwhelm, the cellular machinery responsible for managing misfolded proteins (27), require only that the structure of the aggregate be non-native. The highly stable $\mathrm{H}$-bonded core that appears to exist in the protofibril, based on the HX data presented here, is compatible with toxicity mechanisms requiring specific functional structures.

\section{ACKNOWLEDGMENT}

We acknowledge Bryan M. Saabe and Nityashree Shivaprasad for their assistance with data analysis, and we are also thankful to Kelsey Cook and Al Tuinman for helpful discussions. The MS experiments were performed at the Mass Spectrometry Center in the Department of Chemistry at the University of Tennessee. The molecular EM facility at Harvard Medical School was established by a generous donation from the Giovanni Armenise Harvard Center for Structural Biology and is maintained by funds from NIH Grant GM62580. 


\section{REFERENCES}

1. Kheterpal, I., Zhou, S., Cook, K. D., and Wetzel, R. (2000) Proc. Natl. Acad. Sci. U.S.A. 97, 13597-601.

2. Kheterpal, I., Wetzel, R., and Cook, K. D. (2003) Protein Sci. 12, 635-43.

3. Westermark, P., Benson, M. D., Buxbaum, J. N., Cohen, A. S., Frangione, B., Ikeda, S., Masters, C. L., Merlini, G., Saraiva, M. J., and Sipe, J. D. (2002) Amyloid 9, 197-200.

4. Selkoe, D. J. (1994) Annu. Rev. Neurosci. 17, 489-517.

5. Selkoe, D. J. (1997) Science 275, 630-31.

6. Braak, H., and Braak, E. (1997) Neurobiol. Aging 18, S85-8.

7. Wang, J., Dickson, D. W., Trojanowski, J. Q., and Lee, V. M. (1999) Exp. Neurol. 158, 328-37.

8. Walsh, D. M., Lomakin, A., Benedek, G. B., Condron, M. M., and Teplow, D. B. (1997) J. Biol. Chem. 272, 22364-72.

9. Harper, J. D., and Lansbury, P. T., Jr. (1997) Annu. Rev. Biochem. $66,385-407$

10. Podlisny, M. B., Ostaszewski, B. L., Squazzo, S. L., Koo, E. H., Rydell, R. E., Teplow, D. B., and Selkoe, D. J. (1995) J. Biol. Chem. 270, 9564-70.

11. Walsh, D. M., Klyubin, I., Fadeeva, J. V., Cullen, W. K., Anwyl, R., Wolfe, M. S., Rowan, M. J., and Selkoe, D. J. (2002) Nature 416, 535-9.

12. Hartley, D. M., Walsh, D. M., Ye, C. P., Diehl, T., Vasquez, S., Vassiley, P. M., Teplow, D. B., and Selkoe, D. J. (1999) J. Neurosci. 15, 8876-84.

13. Bucciantini, M., Giannoni, E., Chiti, F., Baroni, F., Formigli, L., Zurdo, J., Taddei, N., Ramponi, G., Dobson, C. M., and Stefani, M. (2002) Nature 416, 507-11.

14. Nilsberth, C., Westlind-Danielsson, A., Eckman, C. B., Condron, M. M., Axelman, K., Forsell, C., Stenh, C., Luthman, J., Teplow, D. B., Younkin, S. G., Naslund, J., and Lannfelt, L. (2001) Nat. Neurosci. 4, 887-93.
15. Lashuel, H. A., Hartley, D. M., Petre, B. M., Wall, J. S., Simon, M. N., Walz, T., and Lansbury, P. T. (2003) J. Mol. Biol. 332, 795-808.

16. Smith, D. L., Deng, Y., and Zhang, Z. (1997) J. Mass Spectrom. $32,135-46$.

17. Hernandez, H., and Robinson, C. V. (2001) J. Biol. Chem. 276 , 46685-88.

18. Wang, S. S.-S., Tobler, S. A., Good, T. A., and Fernandez, E. J. (2003) Biochemistry 42, 9507-14.

19. Nazabel, A., Dos Reis, S., Bonneu, M., Saupe, S. J., and Schmitter, J.-M. (2003) Biochemistry 42, 8852-61.

20. Kheterpal, I., Williams, A., Murphy, C., Bledsoe, B., and Wetzel, R. (2001) Biochemistry 40,11757-67.

21. Harper, J. D., Wong, S. S., Lieber, C. M., and Lansbury, P. T. (1997) Chem. Biol. 4, 119-125.

22. Walsh, D. M., Hartley, D. M., Kusumoto, Y., Fezoui, Y., Condron, M. M., Lomakin, A., Benedek, G. B., Selkoe, D. J., and Teplow, D. B. (1999) J. Biol. Chem. 274, 25945-52.

23. Serio, T. R., Cashikar, A. G., Kowal, A. S., Sawicki, G. J., Moslehi, J. J., Serpell, L., Arnsdorf, M. F., and Lindquist, S. L. (2000) Science 289, 1317-21.

24. Esler, W. P., Stimson, E. R., Jennings, J. M., Vinters, H. V., Ghilardi, J. R., Lee, J. P., Mantyh, P. W., and Maggio, J. E. (2000) Biochemistry 39, 6288-95.

25. Lashuel, H. A., Hartley, D., Petre, B. M., Walz, T., and Lansbury, P. T., Jr. (2002) Nature 418, 291.

26. McCampbell, A., and Fischbeck, K. H. (2001) Nat. Med. 7, 52830 .

27. Bence, N. F., Sampat, R. M., and Kopito, R. R. (2001) Science $292,1552-5$.

BI0357816 